\title{
RENDIMIENTO Y CALIDAD FISICOQUÍMICA EN LÍNEAS EXPERIMENTALES DE Solanum lycopersicum var. cerasiforme CULTIVADAS CON DIFERENTES NIVELES DE CONDUCTIVIDAD ELÉCTRICA
}

\section{YIELD AND PHYSICOCHEMICAL QUALITY IN EXPERIMENTAL LINES OF Solanum lycopersicum var. cerasiforme CULTIVATED WITH DIFFERENT LEVELS OF ELECTRICAL CONDUCTIVITY}

María Teresa Martínez-Damian¹, Juan Enrique Rodríguez-Pérez ${ }^{1}$ Oscar Cruz-Alvarez ${ }^{2 *}$, y María Teresa Beryl Colinas-León ${ }^{1}$

${ }^{1}$ Departamento de Fitotecnia, Universidad Autónoma Chapingo, Carretera México-Texcoco, Km. 38.5, CP 56230, Estado de México, México.

${ }^{2}$ Facultad de Ciencias Agrotecnológicas, Universidad Autónoma de Chihuahua, Avenida Pascual Orozco s/n, Campus 1, Santo Niño, CP 31350, Chihuahua, México.

* Autor de correspondencia E-mail: ocruz@uach.mx

\section{RESUMEN}

El objetivo de esta investigación fue evaluar el rendimiento y calidad fisicoquímica del fruto en siete líneas experimentales avanzadas de tomate cherry, como respuesta a tres niveles $(2,0 ; 2,5 ;$ y 3,0 dS $\mathrm{m}^{-1}$ ) de conductividad eléctrica (CE) de la solución nutritiva de Steiner. El diseño experimental fue completamente al azar con seis repeticiones. Se evaluó el rendimiento, número de frutos por racimo (NFPR), peso promedio de fruto (PPF), color, firmeza, sólidos solubles totales (SST), acidez titulable (AT), vitamina C (VC), fenoles totales (FT), licopeno (Li) y capacidad antioxidante (CA). Los niveles de CE de 2,5 y 3,0 dS m-1 mostraron estadísticamente la mejor firmeza $\left(1,23\right.$ y 1,14 $\left.\mathrm{N} \mathrm{mm}^{-1}\right)$, SST (7,04 y 7,08 ${ }^{\circ}$ Brix), AT (0,49-0,50\% ácido cítrico), VC (13,91 y 8,91 mg $\left.100 \mathrm{~g}^{-1}\right), \mathrm{Li}(9,17$ y 10,75 mg $\left.100 \mathrm{~g}^{-1}\right)$ y CA $\left(45,96-52,21 \mathrm{~mm}^{2}\right.$ TEAC $\left.\mathrm{g}^{-1}\right)$. Entre genotipos, H13-39 fue la de mayor rendimiento y PPF con $9,47 \mathrm{~kg} \mathrm{~m}^{-2}$ y 38,35 g, respectivamente. Adicionalmente L-ChNg1 y L-ChNg2 presentaron los frutos más firmes y H13-26 los de mayor AT y VC. Con excepción de H13-39, L-ChNg1 y L-ChNg2, el resto de genotipos se caracterizaron por su alta CA $\left(55,53-60,60 \mathrm{~mm}^{2}\right.$ TEAC $\left.\mathrm{g}^{-1}\right)$. Sin embargo, H13-39 y L-ChNg1 presentaron los mayores contenidos de Li. La modificación de la CE de la solución nutritiva de Steiner mantuvo sin cambios a los componentes de rendimiento evaluados, pero afectó la calidad fisicoquímica del fruto entre genotipos. Esto permitió observar líneas experimentales sobresalientes y con potencial para su aprovechamiento comercial.

Palabras clave: tomate cherry, manejo agronómico, solución de Steiner, licopeno, capacidad antioxidante

\section{ABSTRACT}

The aim of this research was to evaluate the yield and physicochemical quality of the fruit in seven experimental lines of cherry tomato in response to three levels $\left(2.0,2.5\right.$ and $\left.3.0 \mathrm{dS} \mathrm{m}^{-1}\right)$ of electrical conductivity (EC) of Steiner's nutrient solution. The experimental design was completely randomized with six replicates. Yield, number of fruits per cluster (NFPC), average fruit weight (AFW), color, firmness, total soluble solids (TSS), titratable acidity (TA), vitamin C (VC), total phenols (TF), lycopene (Ly) and antioxidant capacity (AC) were evaluated. EC levels of 2.5 and 3.0 
dS $\mathrm{m}^{-1}$ showed statistically the highest values of firmness $\left(1.23\right.$ and $\left.1.14 \mathrm{~N} \mathrm{~mm}^{-1}\right)$, TSS $(7.04$ and 7.08

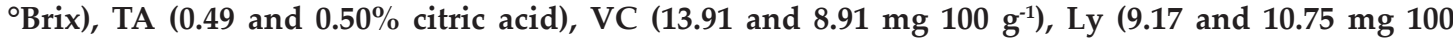
$\mathrm{g}^{-1}$ ) and AC (45.96 and $52.21 \mathrm{~mm}^{2}$ TEAC $\left.\mathrm{g}^{-1}\right)$. Among genotypes, H13-39 recorded the highest yield and AFW, with values of $9.47 \mathrm{~kg} \mathrm{~m}^{-2}$ and $38.35 \mathrm{~g}$, respectively. Additionally, L-ChNg1 and L-ChNg2 presented the fruit with the highest firmness, while H13-26 had those with the highest TA and VC. With the exception of $\mathrm{H13}-39, \mathrm{~L}-\mathrm{ChNg} 1$ and $\mathrm{L}-\mathrm{ChNg} 2$, the rest of the genotypes were characterized by a high AC (55.53-60.60 mm TEAC $\mathrm{g}^{-1}$ ). However, H13-39 and L-ChNg1 had the highest Ly content. The modification of the EC of Steiner's nutrient solution kept the evaluated yield components unchanged, but it affected the physicochemical quality of the fruit among genotypes. This allowed identifying outstanding experimental lines with potential for commercial use.

Key words: cherry tomato, agronomic management, Steiner's solution, lycopene, antioxidant capacity

\section{INTRODUCCIÓN}

El tomate (Solanum lycopersicum) es uno de los cultivos hortícolas de mayor consumo y superficie sembrada en México, donde predomina el tipo saladette ó roma (forma oval) y bola con $51,861.10$ ha (SIAP, 2016); en contraste el tipo cherry o cereza (S. lycopersicum var. cerasiforme) solo representa 3,6\%, donde se destacan los estados de Jalisco, Durango y Guanajuato (FIRA, 2016); su cultivo en la mayoría de los casos se realiza bajo condiciones de invernadero e hidroponía. Este tipo de tomate, de acuerdo con Rodríguez et al. (2009) y Ramya et al. (2016), es una variedad similar pero no idéntica a los ancestros silvestres del tomate domesticado, por lo que la mayoría de los investigadores la consideran la variedad precursora de las formas domesticadas (Ranc et al., 2008), que se cultivan en zonas con diferentes condiciones climáticas (Carrillo-Rodríguez et al., 2012).

En México se puede encontrar gran variedad de genotipos silvestres y semidomesticados de tomate cherry, los cuales se consumen más como fruto que como hortaliza (Rodríguez et al., 2009), sin embargo, por sus características de forma, tamaño, color y sabor, pueden ser ideales en la elaboración de salsa, puré, entre otros tipos de preparaciones (Ramya et al., 2016). Existen varios reportes donde se indica que el tomate cherry presenta concentraciones elevadas de una serie de compuestos bioactivos (licopeno, luteína, $\beta$-caroteno, flavonoides, ácido ascórbico, $\alpha$-tocoferol y vitaminas) (Ranc et al., 2008; Boches et al. 2011;), que en algunos casos supera a los tipos saladette y bola (Carrillo-Rodríguez et al., 2012), lo que le confiere un carácter de alimento nutracéutico (Beckles, 2012). En este sentido, Preczenhak et al. (2014) y Figueroa-Cares et al. (2018) indican que su consumo puede representar una alternativa importante como parte de una alimentación saludable, en el tratamiento de enfermedades neurodegenerativas, cardiovasculares y algunos tipos de cáncer.

Por otro lado, se ha observado que la modificación en la concentración de iones de la solución nutritiva se encuentra correlacionada con el incremento, de compuestos solubles que contribuyen a la calidad intrínseca del fruto (Beckles, 2012; Juárez-López et al., 2013), entre ellos, el contenido de sólidos solubles totales, ácidos orgánicos y azúcares solubles (Boches et al. 2011). Como parte del manejo agronómico, la interacción entre aniones $\left(\mathrm{NO}_{3}{ }^{-}+\mathrm{H}_{2} \mathrm{PO}_{4}+\mathrm{SO}_{4}{ }^{2}\right)$ y cationes $\left(\mathrm{K}^{+}+\mathrm{Ca}^{2+}+\mathrm{Mg}^{2+}\right)$ debe corresponder a la demanda nutrimental del cultivo y con frecuencia son modificadas en cada etapa fenológica de desarrollo (Juárez-López et al., 2013), la cual concluye con la madurez fisiológica del fruto (máxima acumulación de nutrimentos) (Feltrin et al., 2012; Kavitha et al., 2014). Es por ello que el manejo de la conductividad eléctrica de la solución nutritiva debe considerarse como un punto crítico en el sistema de producción de tomate (S. lycopersicum L.) (Krauss et al., 2006; Bertoldi et al., 2008), ya que existen estudios que señalan que valores de conductividad mayores a $6 \mathrm{dS} \mathrm{m}^{-1}$ inducen una marcada diferencia en la absorción entre los nutrimentos, provocando un desbalance entre éstos; no obstante, conductividades menores a $2 \mathrm{dS} \mathrm{m}^{-1}$ se consideran deficientes, sobre todo en zonas de clima templado (Elia et al., 2000; CruzCrespo et al., 2011).

La gran diversidad genética de tomate en México proporciona un alto potencial en los programas de mejoramiento relacionados con calidad de fruto, sin embargo, es necesario contar con información básica de los genotipos utilizados para este fin. Por ello, el objetivo de esta investigación fue evaluar el comportamiento de caracteres de rendimiento y calidad fisicoquímica de fruto de siete líneas experimentales avanzadas de tomate tipo cherry, como respuesta a tres niveles de conductividad $\left(2,0 ; 2,5 ;\right.$ y 3,0 $\left.\mathrm{dS} \mathrm{m}^{-1}\right)$ de la solución de Steiner. 


\section{MATERIALES Y MÉTODOS}

Ubicación del experimento y material vegetal. El experimento se realizó durante los meses de febrero-julio de 2016, en un invernadero tipo "full vent" ubicado en el Campo Experimental "San Martín" de la Universidad Autónoma Chapingo, Estado de México (19²9' 25" N; 9853’23" O) con una altitud de $2240 \mathrm{msnm}$. Se utilizaron siete líneas experimentales avanzadas de tomate tipo cherry (S. lycopersicum var. cerasiforme) (H13-26, H13-29, H13-30, H13-31, H13-39, L-ChNg1 y L-ChNg1) con hábito de crecimiento indeterminado, con diferentes características morfológicas y color de fruto (Tabla 1).

Manejo del cultivo. La siembra se realizó en bandejas de poliestireno expandido con 200 cavidades, utilizando turba (peatmoss) (producto de la descomposición de musgos del genero Sphagnum) como sustrato. A los 35 días se realizó el trasplante en bolsas de polietileno negro rellenas con $13 \mathrm{~kg}$ de tezontle rojo (roca ígnea con alta concentración de dióxido de hierro). Las plantas se condujeron a un solo tallo con una densidad de 3,7 plantas $\mathrm{m}^{-2}$. El suministro de los elementos esenciales para el crecimiento y desarrollo se realizó de acuerdo con los parámetros que establece la solución nutritiva universal de Steiner al 100, 125 y 150\% de su concentración, y complementada con micronutrientes ( $\mathrm{mg} \mathrm{kg-}$ $\left.{ }^{1}\right)$ (fierro (2), manganeso (1), cobre $(0,05)$ y zinc $(0,05))$, con valores de conductividad eléctrica de 2,$0 ; 2,5 ;$ y $3,0 \mathrm{dS} \mathrm{m}^{-1}$, respectivamente (Tabla 2) (Steiner, 1984); el volumen de riego aplicado fue de 0,30-2,5 L planta $^{-1}$ de acuerdo a cada etapa fenológica. El cultivo se llevó hasta el quinto racimo, momento en el cual se despuntó la planta sobre la tercera hoja posterior a dicho racimo. Para la realización de los análisis correspondientes, los frutos que se cosecharon fueron aquellos ubicados entre el primero y quinto racimo en el estado de madurez seis, es decir, cuando el fruto posee $90 \%$ de coloración roja (Choi et al., 1995), la cual coincide con la madurez de consumo. El material se trasladó al laboratorio de fisiología vegetal del Departamento de Fitotecnia de la Universidad Autónoma Chapingo, donde se realizaron las evaluaciones correspondientes.

Tabla 1. Características morfológicas y color de fruto de siete líneas experimentales de tomate tipo cherry (S. lycopersicum var. cerasiforme).

Table 1. Morphological characteristics and fruit color of seven experimental lines of cherry tomato (S. lycopersicum var. cerasiforme).

\begin{tabular}{cclll}
\hline \multirow{2}{*}{$\begin{array}{c}\text { Linea } \\
\text { experimental }\end{array}$} & \multicolumn{4}{c}{ Características del fruto } \\
\cline { 2 - 5 } & Peso $(\mathbf{g})$ & Tamaño & Forma & Color \\
\hline H13-26 & 11,11 & Mediano & Redonda & Amarillo \\
H13-29 & 9,48 & Pequeño & Redonda & Rojo \\
H13-30 & 10,10 & Mediano & Redonda & Rojo \\
H13-31 & 9,72 & Pequeño & Pera & Rojo \\
H13-39 & 38,35 & Gigante & Pera & Rojo \\
L-ChNg1 & 12,84 & Mediano & Pera & Negro \\
L-ChNg2 & 20,78 & Gigante & Pera & Negro \\
\hline
\end{tabular}

*Tamaño del fruto basado en la clasificación de Fernandes et al. (2007).

Tabla 2. Concentración de macroelementos (meq $\left.\mathrm{L}^{-1}\right)$ de las soluciones nutritivas utilizadas en el cultivo de siete líneas experimentales avanzadas de tomate cherry.

Table 2. Concentration of macroelements (meq $\mathrm{L}^{-1}$ ) of the nutrient solutions used in the cultivation of seven advanced experimental lines of cherry tomato.

\begin{tabular}{|c|c|c|c|c|c|c|c|c|c|}
\hline \multirow{2}{*}{$\begin{array}{c}\text { Concentración } \\
(\%) \\
\end{array}$} & \multicolumn{4}{|c|}{ Aniones } & \multicolumn{4}{|c|}{ Cationes } & \multirow[t]{2}{*}{$\mathrm{CE}$} \\
\hline & $\mathrm{NO}_{3}$ & $\mathrm{H}_{2} \mathrm{PO}_{4}$ & $\mathrm{SO}_{4}$ & Total & $\mathrm{K}^{+}$ & $\mathrm{Ca}^{2+}$ & $\mathrm{Mg}^{2+}$ & Total & \\
\hline & 60,0 & 5,00 & 35,00 & 100,00 & 35,00 & 45,00 & 20,00 & 100,00 & \\
\hline 100 & 12,0 & 1,00 & 7,00 & 20,00 & 7,00 & 9,00 & 4,00 & 20,00 & 2,0 \\
\hline 125 & 15,0 & 1,25 & 8,75 & 25,00 & 8,75 & 11,25 & 5,00 & 25,00 & 2,5 \\
\hline 150 & 18,0 & 1,50 & 10,50 & 30,00 & 10,50 & 13,50 & 6,00 & 30,00 & 3,0 \\
\hline
\end{tabular}

${ }^{*} \mathrm{CE}$ : Conductividad eléctrica $\left(\mathrm{dS} \mathrm{m}^{-1}\right)$. 
Diseño experimental. El diseño experimental fue completamente al azar, con seis repeticiones; la unidad experimental consistió de un fruto y las variables evaluadas fueron: rendimiento, número de frutos por racimo, peso promedio de frutos, color (luminosidad, croma y ángulo hue), firmeza, sólidos solubles totales, acidez titulable, vitamina $\mathrm{C}$, fenoles totales, licopeno y capacidad antioxidante.

\section{Variables evaluadas}

Rendimiento (R). Se obtuvo el peso de los frutos (unidad experimental) cosechados mediante una balanza digital portátil OHAUS ${ }^{\circledR}$ con aproximación de $0,01 \mathrm{~g}$. Los datos obtenidos se reportan en $\mathrm{kg} \mathrm{m}^{-2}$.

Número de frutos por racimo (NFPR). Se obtuvo al dividir el total de frutos cosechados entre el total de racimos.

Peso promedio de fruto (PPF). Producto del cociente del valor de rendimiento y el número total de frutos cosechados; el resultado se expresó en gramos $(\mathrm{g})$.

Color. Se determinó de forma directa sobre la epidermis del fruto con colorímetro (X-Rite ${ }^{\circledR}$ serie SP62, Michigan, USA) se tomaron los valores $\mathrm{L}$, a y b en la región ecuatorial de cada fruto. Con estos valores se calculó el ángulo de tono (hue) y la pureza del color (croma) con las fórmulas: Hue $=\arctan \left(b^{*} a^{-1}\right)$; Croma $=\left(a^{2}+b^{2}\right)^{1 / 2}$; y la luminosidad $\mathrm{L}$ obtenido directamente con el colorímetro, los cuales corresponden al espacio de color L*a*b* (Minolta, 1994).

Firmeza (F). La medición se realizó en la zona ecuatorial del fruto mediante un penetrómetro (Chatillón ${ }^{\circledR}$, AMETEK, Florida, USA) provisto de un puntal en forma de cono. La fuerza aplicada hasta la penetración del puntal se expresó en Newtons $\left(\mathrm{N} \mathrm{mm}^{-1}\right)$.

Sólidos solubles totales (SST). Los sólidos solubles totales ( ${ }^{\circ}$ Brix) se cuantificaron con un refractómetro digital portátil PAL-1 ${ }^{\circledR}$ (ATAGO, Washington, USA) el cual utiliza una escala de $0-53^{\circ}$, y para realizar la medición se colocó una gota de jugo del fruto en la pantalla del refractómetro procediendo a tomar la lectura.

Acidez titulable (AT). Se determinó de acuerdo a la metodología propuesta por la AOAC (AOAC, 1990), con $20 \mathrm{~g}$ de pulpa que fue neutralizada con $\mathrm{NaOH} 0,1 \mathrm{~N}$, utilizando fenolftaleína al 1\% como indicador. Los resultados se reportaron en \% de ácido cítrico (ácido orgánico prevaleciente).

Vitamina C (VC). Se estimó de acuerdo con el método propuesto por Jacota y Dani (1982) con ligeras modificaciones. Se homogeneizaron $4 \mathrm{~g}$ de fruto en $5 \mathrm{~mL}$ de ácido tricloroacético al $20 \%$, la mezcla se dejó reposar por $5 \mathrm{~min}$ en frio (hielo) y se centrifugó durante $20 \mathrm{~min}$ a 15000 rpm a $4{ }^{\circ} \mathrm{C}$. Posteriormente se tomó una alícuota de $0,2 \mathrm{~mL}$ del sobrenadante de cada muestra y se adicionaron $1,8 \mathrm{~mL}$ de agua destilada y $0,2 \mathrm{~mL}$ del reactivo Folin-Ciocalteu al $10 \%$. La mezcla se agitó vigorosamente y se dejó en reposo por 10 min para posteriormente realizar la lectura de absorbancia a $760 \mathrm{~nm}$. La concentración se expresó en mg $100 \mathrm{~g}^{-1}$ de peso fresco mediante una curva estándar de ácido ascórbico.

Fenoles totales (FT). La determinación de fenoles totales se llevó a cabo mediante el método de Folin y Ciocalteu planteado por Waterman y Mole (1994), con las siguientes modificaciones: se tomaron $300 \mu \mathrm{L}$ del extracto etanólico $(1,0 \mathrm{~g}$ de pulpa en $5 \mathrm{~mL}$ de etanol, homogeneizados con $24 \mathrm{~h}$ de reposo) a los que se le adicionaron $8,0 \mathrm{~mL}$ de agua destilada, $0,5 \mathrm{~mL}$ del reactivo de Folin y Ciocalteu, respectivamente, y se agitó; finalmente se agregaron $1,5 \mathrm{~mL}$ de solución de $\mathrm{Na}_{2} \mathrm{CO}_{3}$ al $20 \%$ a cada muestra y se volvió a agitar, dejando reposar durante $2 \mathrm{~h}$ bajo condiciones de oscuridad. Se tomó la lectura de absorbancia a 760 $\mathrm{nm}$. Los resultados se expresaron en $\mathrm{mg} 100 \mathrm{~g}^{-1} \mathrm{de}$ peso fresco, de acuerdo a una curva estándar de ácido tánico.

Licopeno (Li). La determinación se realizó empleando el método modificado de Sadler et al. (1990). Se homogeneizaron $20 \mathrm{~g}$ de pulpa con agua destilada, la mezcla obtenida fue colocada en un frasco envuelto en papel aluminio y secadas a $38^{\circ} \mathrm{C}$. Se colocó $0,1 \mathrm{~g}$ de la pasta en tubos de ensayo cubiertos con papel aluminio, se les adicionaron $30 \mathrm{~mL}$ de una mezcla de hexano/ etanol/acetona en la proporción 2:1:1 y se agitaron por $10 \mathrm{~min}$. Posteriormente, se adicionaron 18 $\mathrm{mL}$ de agua destilada y se agitó durante $5 \mathrm{~min}$ hasta la separación de la fase acuosa y orgánica. Con matraces de separación se tomó y midió el volumen de la fase orgánica a la cual se le midió el valor de absorbancia a $470 \mathrm{~nm}$. La cuantificación se realizó mediante la fórmula de Inbaraj et al. (2008) y los resultados se expresaron en mg 100 $\mathrm{g}^{-1}$ de peso fresco.

Capacidad antioxidante (CA). Se realizó de acuerdo con el método ABTS (2,2'azinobis (3-etilbenzotiazolin-6-ácido sulfónico) descrito 
por Ozgen et al. (2006), para lo cual el radical $\mathrm{ABTS}^{*}$ se formó tras la reacción de ABTS $(7 \mathrm{mM})$ con persulfato potásico $(2,45 \mathrm{mM}$, concentración final) incubados a temperatura ambiente $y$ en oscuridad durante $24 \mathrm{~h}$. Una vez formado el radical $\mathrm{ABTS}^{*+}$ este se diluyó con PBS (solución buffer de acetato de sodio) ( $\mathrm{pH} 4,5)$ hasta obtener un valor de absorbancia de 0,7 $\pm 0,1$ a $734 \mathrm{~nm}$ (longitud de máxima absorción). Para el ensayo, se mezclaron $3,9 \mathrm{ml}$ de la solución $\mathrm{ABTS}^{\bullet+}$ y 100 $\mu \mathrm{l}$ de extracto de la muestra, dejándose reposar por $2 \mathrm{~h}$, donde la lectura de absorbancia se realizó a $734 \mathrm{~nm}$. Los resultados se expresan en TEAC (actividad antioxidante equivalente a Trolox).

Análisis estadístico. Se realizó un análisis de varianza (ANOVA) y comparación de medias de Tukey $(\mathrm{P} \leq 0,05)$, en las que se empleó el programa de análisis estadístico Statistical Analysis System (SAS, 2002).

\section{RESULTADOS Y DISCUSIÓN}

Rendimiento. El rendimiento no fue afectado al modificar la CE $\left(2,0 ; 2,5 ;\right.$ y $\left.3,0 \mathrm{dS} \mathrm{m}^{-1}\right)$ de la solución nutritiva de Steiner, cuyos valores fluctuaron de 4,38 a 4,99 $\mathrm{kg} \mathrm{m}^{-2}$ (Tabla 3). Al respecto Bertoldi et al. (2008) reportan un comportamiento similar para la variedad de tomate cherry 'Mascote' cultivada con tres niveles de conductividad eléctrica $\left(3,0 ; 6,0 ; y\right.$ 9,0 dS $\left.\mathrm{m}^{-1}\right)$, pero con valores de rendimiento más bajos de 2,03; 2,57; y 3,09 $\mathrm{kg} \mathrm{m}^{-2}$, respectivamente. Por su parte, Feltrin et al. (2012) encontraron incrementos significativos de 2,82 a 3,07 $\mathrm{kg} \mathrm{m}^{-2}$ en tomate cherry 'Cascade' con conductividades de 1,5 y $2,0 \mathrm{dS} \mathrm{m}^{-1}$, mientras que conductividades mayores $\left(2,5\right.$ a $\left.3,5 \mathrm{dS} \mathrm{m}^{-1}\right)$ el rendimiento disminuyó de 3,07 a 2,20 $\mathrm{kg} \mathrm{m}^{-2}$.

Alcompararentregenotipos(Tabla3)seencontró que H13-39 de fruto de tamaño gigante presentó un rendimiento $\left(8,75 \mathrm{~kg} \mathrm{~m}^{-2}\right)$ significativamente más alto que el resto de materiales evaluados $(3,29$ a $4,78 \mathrm{~kg} \mathrm{~m}^{-2}$ ). Estos resultados concuerdan con lo reportado en seis poblaciones de tomate cherry por Boada-Higuera et al. (2010), con valores de 1,57 a $8,0 \mathrm{~kg} \mathrm{~m}^{-2}$. Adicionalmente Parra-Gómez et al. (2016) en una evaluación de líneas de una cruza interespecífica de tomate, indican la existencia de una alta correlación entre el rendimiento con el peso promedio de fruto, longitud y diámetro de fruto, así como el número total de frutos, por lo que constituyen caracteres muy importantes de selección en mejoramiento genético. Los materiales analizados en este trabajo presentan datos de rendimiento más altos que algunos materiales silvestres tipo cereza, sin embargo, este parámetro agronómico no supera al reportado en cultivares comerciales.

Número de frutos por racimo (NFPR). Valores de CE de 2,0, 2,5 y 3,0 $\mathrm{dS} \mathrm{m}^{-1}$, no provocaron variaciones en el NFPR (Tabla 3 ). Un resultado

Tabla 3. Efecto del genotipo y la conductividad de la solución nutritiva sobre los componentes del rendimiento y la calidad fisicoquímica en tomate cherry.

Table 3. Effect of genotype and conductivity of the nutrient solution on the yield components and physicochemical quality of cherry tomato.

\begin{tabular}{lccrrcc}
\hline Genotipo & R & NFPR & PPF & \multicolumn{3}{c}{ Color } \\
\cline { 5 - 7 } & & & & $\mathbf{L}$ & $\mathbf{C}$ & $\mathbf{H}$ \\
\hline H13-26 & $3,59 \mathrm{~b}$ & $17,42 \mathrm{~b}$ & $11,11 \mathrm{c}$ & $48,89 \mathrm{a}$ & $44,86 \mathrm{a}$ & $71,75 \mathrm{a}$ \\
H13-29 & $4,79 \mathrm{~b}$ & $27,27 \mathrm{a}$ & $9,48 \mathrm{c}$ & $41,22 \mathrm{~b}$ & $36,38 \mathrm{~b}$ & $49,87 \mathrm{dc}$ \\
H13-30 & $5,17 \mathrm{~b}$ & $28,22 \mathrm{a}$ & $10,10 \mathrm{c}$ & $39,77 \mathrm{~b}$ & $32,17 \mathrm{cb}$ & $50,94 \mathrm{c}$ \\
H13-31 & $4,34 \mathrm{~b}$ & $24,78 \mathrm{a}$ & $9,72 \mathrm{c}$ & $38,59 \mathrm{~b}$ & $31,26 \mathrm{~cd}$ & $49,58 \mathrm{dc}$ \\
H13-39 & $9,47 \mathrm{a}$ & $13,11 \mathrm{cb}$ & $38,35 \mathrm{a}$ & $42,46 \mathrm{~b}$ & $33,67 \mathrm{cb}$ & $50,77 \mathrm{c}$ \\
L-ChNg1 & $3,56 \mathrm{~b}$ & $15,04 \mathrm{cb}$ & $12,84 \mathrm{c}$ & $38,67 \mathrm{~b}$ & $30,18 \mathrm{~cd}$ & $47,76 \mathrm{~d}$ \\
L-ChNg2 & $3,88 \mathrm{~b}$ & $10,27 \mathrm{c}$ & $20,78 \mathrm{~b}$ & $39,10 \mathrm{~b}$ & $26,89 \mathrm{~d}$ & $53,65 \mathrm{~b}$ \\
DMSH & 4,39 & 6,70 & 6,66 & 4,55 & 4,62 & 2,52 \\
\hline CE & & & & & & \\
\hline 2,0 & $5,41 \mathrm{a} \S$ & $21,70 \mathrm{a}$ & $15,97 \mathrm{a}$ & $40,67 \mathrm{a}$ & $34,72 \mathrm{a}$ & $53,75 \mathrm{a}$ \\
2,5 & $4,74 \mathrm{a}$ & $18,30 \mathrm{a}$ & $15,92 \mathrm{a}$ & $42,31 \mathrm{a}$ & $33,45 \mathrm{a}$ & $53,45 \mathrm{a}$ \\
3,0 & $4,77 \mathrm{a}$ & $18,34 \mathrm{a}$ & $16,28 \mathrm{a}$ & $40,74 \mathrm{a}$ & $32,72 \mathrm{a}$ & $53,21 \mathrm{a}$ \\
DMSH & 1,31 & 3,44 & 3,42 & 2,35 & 2,39 & 1,29 \\
\hline
\end{tabular}

R: Rendimiento $\left(\mathrm{kg} \mathrm{m}^{-2}\right)$; NFPR: Número de frutos por racimo; PPF: Peso promedio de fruto (g); L: Luminosidad; C: Cromaticidad; H: ( $\left.{ }^{\circ} \mathrm{Hue}\right)$; CE: Conductividad eléctrica $\left(\mathrm{dS} \mathrm{m}^{-1}\right)$. DMSH: diferencia mínima significativa honesta. ${ }^{\S}$ Medias con la misma letra dentro de la columna no difieren significativamente de acuerdo con la prueba de Tukey $(p \leq 0,05)$. 
similar fue reportado por Bertoldi et al. (2008). Por otro lado, se indican incrementos en el número de frutos por racimo de 151 a 172 por Feltrin et al. (2012) en la variedad de tomate cherry 'Sweet Million', cuando la conductividad pasó de 1,5 a 2,0 $\mathrm{dS} \mathrm{m}^{-1}$; estos mismos autores también señalan que al utilizar valores de conductividad eléctrica 2,5 a $3,5 \mathrm{dS} \mathrm{m}^{-1}$, esta variable disminuyó de 184 a 141 frutos por planta, lo cual sugiere que el número de frutos en este tipo de tomate se encuentra asociada en gran medida con la susceptibilidad del genotipo a la variación de la CE (Boches et al., 2011).

Entre genotipos, las líneas experimentales H13-29, H13-30 y H13-31 presentaron mayor NFPR $(24,7$ a 28,2$)$ en relación al resto de los materiales analizados $(10,27$ a 17,42$)$ (Tabla 3). Carrillo-Rodríguez et al. (2012) reportaron para 49 y 20 accesiones de tomates cereza silvestres y semidomesticados colectados en México, un número de frutos menor $(4,1$ a 9,3 y 7,0 a 11,0 , respectivamente). Por su parte, Ceballos-Aguirre et al. (2012) indicaron mayor variación para este parámetro con valores de 3,6 a 34 frutos por racimo, en 30 poblaciones tipo cereza en Colombia. La gran variabilidad en el número de frutos para este tipo de tomate se encuentra relacionada directamente con el grado de domesticación o selección, asi como por las condiciones edafoclimáticas prevalecientes durante el crecimiento y desarrollo de la planta, ya que de acuerdo con Rodríguez et al. (2009) y Carrillo-Rodríguez y Chávez-Servia (2010), al ser una especie de fruto pequeño, el número de frutos puede ser un buen indicador sobre origen silvestre del material analizado.

\section{Color}

Luminosidad. La utilización de diferentes niveles de CE de la solución nutritiva de Steiner no representó una variación en el comportamiento de la luminosidad del color del fruto, donde se encontró un rango de valores de 40,67 y 42,31 (Tabla 3). En relación con estos resultados, datos similares de brillantez son reportados por Cruz-Crespo y Sandoval-Villa (2011) en frutos de tomate saladette 'Charleston' cultivados con concentraciones de la solución de Steiner de $0,50,75$ y $100 \%$. Sin embargo, en un trabajo realizado en la variedad de tomate 'Durinta', se indicaron valores de 70,1 a 72,9, con intervalos de variaciones de conductividad más elevados a los estudiados en este trabajo $\left(2,8\right.$ a $\left.7,7 \mathrm{dS} \mathrm{m}^{-1}\right)(\mathrm{Kim}$ et al., 2008).

Al comparar entre genotipos (Tabla 4), se puede observar que la línea H13-26 de fruto amarillo presentó valores de luminosidad significativamente más altos $(48,89)$ que todos los demás genotipos, los cuales exhibieron brillantez estadísticamente similar $(38,59$ a 42,46). Valores similares de brillantez entre 33,49 y 45,2 fueron reportados por Preczenhak et al. (2014) en 64 genotipos de tomate cherry (S. lycopersicum var. cerasiforme) provenientes de Brasil, siendo el genotipo de fruto color amarillo oro el que presentó el valor más alto. Sin embargo, Csambalik et al. (2014) encontraron brillantez de fruto más bajas $(30,47$ y 33,91) en seis genotipos de tomate cherry. De acuerdo con Preczenhak et al. (2014) para el tomate cherry el brillo ideal de fruto está entre 38,0 y 40,0; por lo que los genotipos H13-30, H13-31, L-ChNg1 y L-ChNg2 $(38,6$ a 39,7$)$ presentaron características deseables para este carácter de calidad.

Croma. La pureza de color del fruto no presentó valores significativos en ninguno de los niveles de CE evaluados, cuyos valores fluctuaron entre 32,72 y 34,72 (Tabla 3). CruzCrespo y Sandoval-Villa (2011) señalaron para frutos de tomate 'Charleston' producidos con cuatro concentraciones de la solución de Steiner $(0,50,75$ y $100 \%)$, un rango de datos de 21,86 a 22,43. Sin embargo, Kim et al. (2008) encontraron incrementos significativos de croma $(31,9$ a 34,4$)$ de frutos de 'Durinta' a niveles de conductividad más altos a los evaluados en este trabajo $(2,8$ a 7,7 $\mathrm{dS} \mathrm{m}^{-1}$ ).

La línea H13-26 de fruto amarillo presentó significativamente el valor de croma más alto $(44,86)$, en contraste L-ChNg2 con 26,9 fue la de menor intensidad de color (Tabla 3). Preczenhak et al. (2014) indicaron valores estadísticamente menores $(16,26$ y 29,40$)$ para 64 accesiones de tomate tipo cherry (S. lycopersicum var. cerasiforme) cosechadas en Brasil, donde de forma coincidente las de fruto amarillo presentaron los valores más altos. Resultados similares son señalados por Csambalik et al. (2014) quienes encontraron valores de cromaticidad entre 21,12 y 26,09 en seis genotipos tipo cherry cultivados en Turquía. Según Preczenhak et al. (2014), valores de croma cercanos a cero representan colores combinados y opacos, mientras que valores próximos a 60 indican colores puros y vivaces; en el presente estudio, los materiales analizados presentaron intensidades de color aceptables, por lo que podrían ser susceptibles a ser aprovechados comercialmente o para su uso en programas de mejoramiento genético en la que se incluya este componente de color.

Ángulo hue. Con un comportamiento similar a la cromaticidad, la tonalidad de color no presentó diferencias significativas y sus valores 
Tabla 4. Efecto del genotipo y la conductividad de la solución nutritiva sobre la calidad fisicoquímica en frutos de tomate cherry.

Table 4. Effect of genotype and conductivity of the nutrient solution on the physicochemical quality of cherry tomato fruits.

\begin{tabular}{llllc}
\hline Genotipo & F & SST & AT & VC \\
\hline H13-26 & $0,88 \mathrm{c}^{\S}$ & $6,84 \mathrm{c}$ & $0,64 \mathrm{a}$ & $0,64 \mathrm{a}$ \\
H13-29 & $1,02 \mathrm{c}$ & $7,03 \mathrm{bc}$ & $0,52 \mathrm{cb}$ & $10,56 \mathrm{~cd}$ \\
H13-30 & $1,07 \mathrm{bc}$ & $7,62 \mathrm{ba}$ & $0,54 \mathrm{~b}$ & $12,94 \mathrm{cb}$ \\
H13-31 & $0,98 \mathrm{c}$ & $8,14 \mathrm{a}$ & $0,53 \mathrm{~b}$ & $0,53 \mathrm{~b}$ \\
H13-39 & $1,29 \mathrm{ba}$ & $5,62 \mathrm{~d}$ & $0,46 \mathrm{c}$ & $4,09 \mathrm{e}$ \\
L-ChNg1 & $1,34 \mathrm{a}$ & $7,40 \mathrm{bc}$ & $0,35 \mathrm{~d}$ & $9,13 \mathrm{~d}$ \\
L-ChNg2 & $1,44 \mathrm{a}$ & $6,03 \mathrm{~d}$ & $0,34 \mathrm{~d}$ & $5,43 \mathrm{e}$ \\
DMSH & 0,24 & 0,59 & 0,06 & 2,67 \\
\hline CE & & & & \\
\hline 2,0 & $1,07 \mathrm{~b}$ & $6,74 \mathrm{~b}$ & $0,46 \mathrm{~b}$ & $9,05 \mathrm{~b}$ \\
2,5 & $1,23 \mathrm{a}$ & $7,04 \mathrm{ba}$ & $0,49 \mathrm{a}$ & $13,91 \mathrm{a}$ \\
3,0 & $1,14 \mathrm{ba}$ & $7,08 \mathrm{a}$ & $0,50 \mathrm{a}$ & $8,93 \mathrm{~b}$ \\
DMSH & 0,12 & 0,31 & 0,03 & 1,38 \\
\hline
\end{tabular}

F: Firmeza $\left(\mathrm{N} \mathrm{mm}^{-1}\right)$; SST: Solidos solubles totales ( ${ }^{\circ}$ Brix); AT: Acidez titulable (\% ácido cítrico); VC: Vitamina C

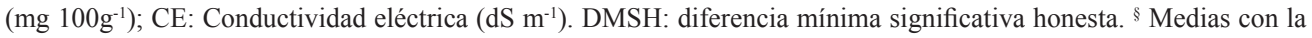
misma letra dentro de la columna no difieren significativamente de acuerdo con la prueba de Tukey $(\mathrm{p} \leq 0,05)$.

fluctuaron entre 53,75 y $53,21^{\circ}$ (Tabla 3). Estos resultados son similares a 58,0 y $62,95^{\circ}$ reportadas en frutos de tomate 'Charleston' cultivados con tres concentraciones (50, 75 y 100\%) de la solución de Steiner (Cruz-Crespo y Sandoval-Villa, 2011). En contraste, Kim et al. (2008) al ajustar los valores de CE de 2,8 a 7,7 dS m-1, encontraron una disminución significativa en la tonalidad de color del fruto de 59,7 a $53,5^{\circ}$ en frutos de tomate 'Durinta'.

Entre genotipos, la línea experimental H1326 fue la de mayor tonalidad de color $\left(71,7^{\circ}\right)$ al superar significativamente a los genotipos de fruto rojo (H13-29, H13-30, H13-31 y H13-39), los cuales presentaron valores entre 49,5 y $50,9^{\circ}$ (Tabla 3). En este sentido, Csambalik et al. (2014) reportaron valores similares $\left(44,63\right.$ y $\left.58,34^{\circ}\right)$ en frutos rojos de seis cultivares de tomate cherry producidos en Hungría. El comportamiento observado en H13-26 de fruto amarillo concuerda con lo reportado por Preczenhak et al. (2014) para el genotipo 2091-44 también de fruto amarillo $\left(85,76^{\circ}\right)$.

Los valores de tonalidad de color ( ${ }^{\circ}$ hue) encontrados en esta investigación, específicamente en los genotipos con frutos de epidermis roja superan los 35 a $40^{\circ}$ hue reportados por Pandurangaiah et al. (2016) para frutos comerciales cosechados con similar grado de madurez, sino más bien son coincidentes con el dato de $48^{\circ}$ que corresponde a un tono rojonaranja que también son reportados por estos mismos autores.
Firmeza. La modificación en los valores de la CE de 2,0 a $2,5 \mathrm{dS} \mathrm{m}^{-1}$ modificó $(\mathrm{P} \leq 0,05)$ el comportamiento de la firmeza del fruto en $14,9 \%\left(1,07\right.$ a 1,23 $\left.\mathrm{N} \mathrm{mm}^{-1}\right)$ (Tabla 4). Resultados similares fueron reportados por Juárez-López et al. (2013) en cuatro genotipos nativos de tomate cherry cuyos valores mostraron un incremento de 6,9 a $7,8 \mathrm{~N} \mathrm{~mm}^{-1}(10.8 \%)$, al elevar la concentración de la solución de Steiner de 50 a $100 \%$. Por su parte, Wang et al. (2007) indicaron que al elevar la concentración de nitrógeno de 2,2 a 9,0 mmol $\mathrm{L}^{-1}$ mejoró la firmeza de fruto de 4,5 a $5,9 \mathrm{kPa}$ en el cultivar 'Chuanzhu'. Adicionalmente estos mismos autores, señalaron una disminución de 5,5 a $4,8 \mathrm{kPa}$, cuando los frutos se cultivaron con valores de concentración de 18,0 y $36,0 \mathrm{mmol} \mathrm{L}^{-1}$ de nitrógeno, lo cual sugiere un efecto antagónico entre el crecimiento vegetativo y el desarrollo adecuado de características de calidad del fruto como la firmeza del fruto y la concentración de sólidos solubles.

Entre los diversos materiales evaluados se observó que las líneas experimentales de fruto negro (L-ChNg2, L-ChNg1) y fruto rojo (H13-39), presentaron valores de firmeza sobresalientes (1,29 y 1,44 $\left.\mathrm{N} \cdot \mathrm{mm}^{-1}\right)$ (Tabla 4) con relación a H13-26, H13-29, H13-30 y H13-31, cuyos valores fluctuaron entre 0,88 y $1,07 \mathrm{~N} \cdot \mathrm{mm}^{-1}$. Al realizar la búsqueda de trabajos realizados en este tipo de tomate, se encontró gran disparidad entre los diferentes reportes, como el realizado en cuatro genotipos nativos de tomate cherry por JuárezLópez et al. (2013) $\left(5,8\right.$ a 9,3 N.mm $\left.{ }^{-1}\right)$. Por otro 
lado, de acuerdo con Figueroa-Cares et al. (2018), el fruto de tomate requiere un mínimo de firmeza $\left(1,46 \mathrm{~N} \mathrm{~mm}^{-1}\right)$ para su comercialización como producto en fresco, lo que significa que, para el grado de madurez analizado en este trabajo, todos los materiales evaluados no satisfacen este requerimiento. Sin embargo, si se considera el tamaño y las características morfológicas de este tipo de tomate, la cosecha, empaque y comercialización se realiza por racimos completos; por lo que se reduce el daño mecánico y pérdida de valor económico.

Sólidos solubles totales. Se encontraron diferencias significativas en la concentración de SST de los frutos manejados a 2,5 y 3,0 dS $\mathrm{m}^{-1}$ con valores de 7,04 y 7,08 ${ }^{\circ}$ Brix, respectivamente (Tabla 4). Feltrin et al. (2012) indicaron un incremento lineal significativo respecto a la acumulación de sólidos solubles totales $(5,8$, $6,7,7,6$ y $7,9^{\circ}$ Brix), en frutos de tomate cherry 'Sweet Million' manejados con solución nutritiva y valores de conductividad eléctrica $(1,5,2,0,2,5$ y $3,0 \mathrm{dS} \mathrm{m}^{-1}$ ) similares a los de este trabajo. Por su parte, al incrementar la concentración de la solución de Steiner de 50 a $100 \%$ en el manejo de cuatro variedades de tomate cherry, JuárezLópez et al. (2013) reportaron un incremento de $5,89 \%$ en los valores de éste parámetro, cuya variación fue de 8,5 a 9,0 ${ }^{\circ}$ Brix, lo que sugiere en parte un aumento en el sabor y una baja en el contenido de agua (Beckles, 2012), como una de las consecuencias del desarrollo del proceso de maduración del fruto.

La mayor acumulación de SST entre las diversas líneas experimentales se presentó en H13-30 y H13-31, con medias de 7,62 y 8,14 ${ }^{\circ}$ Brix (Tabla 4), sin embargo, H13-29 mostró un comportamiento similar con respecto a H13-30. Concentraciones más bajas de SST son reportadas por Kavitha et al. (2014) para 13 líneas experimentales provenientes de India y variedades comerciales cultivadas en España, con valores de 4,1 a 9,5 y de 5,47 y 8,71 ${ }^{\circ}$ Brix, respectivamente. En contraste, Ceballos y Vallejo (2012) al evaluar el contenido de sólidos solubles totales en 30 colectas de tomate, donde incluyeron una variedad comercial ('Sweet Million'), indican un rango de valores entre 4,04 y $6,7^{\circ}$ Brix; comportamiento interesante debido al valor detectado para esta variedad $\left(4,91^{\circ} \mathrm{Brix}\right)$, la cual no supera a lo observado en los materiales analizados en este trabajo.

Acidez titulable (AT). La acidez del fruto mostró un incremento significativo de 0,46 a $0,50 \%$ cuando se modificó la CE de 2,5 a 3,0 dS $\mathrm{m}^{-1}$ (Tabla 4). Juárez-López et al. (2013) describen una variación entre 0,70 y $0,73 \%$ de ácido cítrico en cuatro genotipos de tomate cherry cuando se cambió la concentración de la solución de Steiner de 75 a $100 \%$. Con una conductividad eléctrica similar, Feltrin et al. (2012), reportaron para frutos tipo cereza del cultivar 'Cascade' valores de 0,33 a 0,34 mg ácido cítrico $100 \mathrm{~g}^{-1}$, no obstante, también reportaron datos de 0,29 y $0,33 \mathrm{mg}$ ácido cítrico $100 \mathrm{~g}^{-1}$ cuando se usó un rango menor de conductividad (1,5 a 2,5 dS m-1 $)$, comportamiento que se asocia directamente con un menor flujo de agua a través del xilema durante la maduración del fruto (Elia et al., 2000).

Entre genotipos, se destacó H13-26 que presentó frutos con valores de porcentaje de ácido cítrico de 0,64 , cuya variación máxima representó $53 \%$ con respecto a L-ChNg1 y L-ChNg2 de fruto negro $(0,34$ y 0,35$)$ (Tabla 4$)$. Un rango de valores similares de ácido cítrico de 0,35 a $0,60 \%$ fueron reportados por Kavitha et al. (2014). En este sentido, un valor alto producto de la relación entre acidez y contenido de sólidos solubles totales es un claro indicador de calidad (Feltrin et al., 2012), que se asocia directamente con frutos de sabor agradable.

Vitamina C (VC). La concentración de ácido ascórbico fue afectada $(\mathrm{P} \leq 0,05)$ de manera puntual con una CE de 2,5 dS $\mathrm{m}^{-1}$ en la solución nutritiva, con valores de 13,91 mg $100 \mathrm{~g}^{-1}$ (Tabla 4). Variaciones entre los niveles de conductividad eléctrica de 1,0 a 2,0 y de 3,0 a $9,0 \mathrm{dS} \mathrm{m}^{-1}$ manejadas en el cultivo de variedades de tomate cherry permitieron obtener contenidos de vitamina $C$ de 31,7 a 32,9 y de 16,1 a $28,9 \mathrm{mg} 100 \mathrm{~g}^{-1}$, sin observar diferencia entre los rangos evaluados (JuárezLópez et al., 2013), lo cual también coincide con lo señalado para tomate tipo saladette de crecimiento indeterminado por Fanasca et al. (2006) quienes reportaron valores de 37 a $54 \mathrm{mg}$ $100 \mathrm{~g}^{-1}$. Por su parte, Elia et al. (2000) reportaron que la variación de la conductividad eléctrica de 2,0 a 4,0 dS m$~^{-1}$ fue un factor a considerar en el incremento de $25.2 \%$ de vitamina en frutos del cultivar 'Naomi'.

En esta evaluación, se encontró que los frutos pequeños provenientes de H13-31 presentaron el contenido más alto de vitamina $C(17,9 \mathrm{mg} 100$ $\mathrm{g}^{-1}$ ) (Tabla 4). Al respecto, los niveles de vitamina $\mathrm{C}$ encontrados fueron similares a los descritos (12,79 a 21,40 mg ác. asc. $\left.100 \mathrm{~g}^{-1}\right)$ por FigueroaCares et al. (2018) en genotipos nativos de tomate tipo cereza, y fueron superiores a lo descrito por Leiva-Brondo et al. (2012) en seis genotipos cherry cultivados en España (7,4 y 14,4 mg 100 $\left.\mathrm{g}^{-1}\right)$. No obstante, autores como Guil-Guerrero y Rebolloso-Fuentes (2009) en un estudio donde se evaluó la composición nutrimental y capacidad antioxidante en ocho variedades de 
tomate cultivadas en España, indicaron un rango de valores de vitamina C de 39 a $263 \mathrm{mg} 100 \mathrm{~g}$ peso fresco, los cuales superan por mucho a lo observado en este trabajo, lo cual se puede asociar por un lado al grado de selección para este carácter (Ranc et al., 2008), así como por el nivel de sombreado al que fueron sometidos durante la etapa final de maduración, ya que según LeivaBrondo et al. (2012) este compuesto muestra alta susceptibilidad a degradarse por efecto de la luz.

Fenoles totales (FT). El contenido de FT se mantuvo sin cambios en los niveles de CE utilizados, con valores que fluctuaron entre 3,36 y $3,58 \mathrm{mg} 100 \mathrm{~g}^{-1}$. En este sentido, Bertoldi et al. (2008) describieron variaciones no significativas

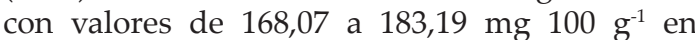
frutos de tomate cherry 'Mascote', producidos con tres niveles de conductividad eléctrica en la solución de Steiner $\left(3,0,6,0\right.$ y 9,0 dS $\left.\mathrm{m}^{-1}\right)$. Por su parte, Krauss et al. (2006) al utilizar niveles de conductividad más altos $\left(6,5\right.$ a 13,5 dS m $\left.\mathrm{m}^{-1}\right)$, indicaron un incremento significativo de fenoles totales en 'Durinta' (28,5 a 48,1 mg $\left.100 \mathrm{~g} \mathrm{~g}^{-1}\right)$, donde las diferencias entre resultados puede estar asociado con la naturaleza del disolvente de extracción, debido a la presencia de compuestos antioxidantes con naturaleza química y polaridad contrastante que pueden o no ser solubles en un disolvente particular (Guil-Guerrero y RebollosoFuentes, 2009).

Entre los frutos evaluados, los correspondientes a H13-26 de color amarillo presentaron la mayor concentración de FT (4,34 mg $\left.100 \mathrm{~g}^{-1}\right)$, el cual superó estadísticamente a H13-30, H13-39, L-ChNg1 y L-ChNg2, cuyo contenido fluctuó entre 2,57 y 3,75 mg $100 \mathrm{~g}^{-1}$ (Tabla 4). Noor Atiqah et al. (2014) al evaluar materiales de tomate cherry amarillo y de tipo saladette de fruto rojo, reportaron que los genotipos de fruto amarillo mostraron los niveles más altos de fenoles totales

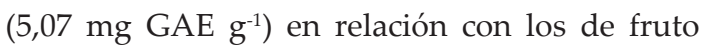
rojo $\left(4,28 \mathrm{mg} \mathrm{GAE} \mathrm{g}^{-1}\right)$. Por otro lado, Kavitha et al. (2014) indicaron valores que superaron a los encontrados en este estudio $(48,2$ a $73,4 \mathrm{mg}$ GAE $100 \mathrm{~g}^{-1}$ de peso fresco) en frutos de 13 líneas de tomate cherry cultivados en India. Es importante mencionar que además de su alta concentración de fenoles totales, la línea experimental H13-26 se destaca por su tamaño medio, forma redonda y color amarrillo; caracteres de selección muy atractivos para el desarrollo de nuevos materiales de uso comercial, ya que de acuerdo con Boches et al. (2011) este tipo de características generan un reducido arrastre de alelos desfavorables para otros rasgos.

Licopeno (Li). Niveles de CE entre 2,0 y 3,0 dS $\mathrm{m}^{-1}$ permitieron incrementar significativamente la presencia de licopeno de 7,89 a 10,75 mg 100 $\mathrm{g}^{-1}$ (Tabla 5), comportamiento altamente asociado con la composición y concentración de la solución nutritiva, así como por efecto del grado de mejoramiento del material vegetal evaluado (Fanasca et al., 2006). Resultados similares (42,0 a $49,4 \mathrm{mg} 100 \mathrm{~g}^{-1}$ ) fueron reportados por Juárez-

Tabla 5. Efecto del genotipo y la conductividad eléctrica de la solución nutritiva sobre la calidad fisicoquímica en frutos de tomate cherry.

Table 5. Effect of genotype and electrical conductivity of the nutrient solution on the physicochemical quality of cherry tomato fruits.

\begin{tabular}{llcc}
\hline Genotipo & FT & Li & CA \\
\hline H13-26 & $4,34 \mathrm{a}^{\S}$ & $1,38 \mathrm{c}$ & $58,85 \mathrm{a}$ \\
H13-29 & $3,80 \mathrm{~b}$ ac & $9,34 \mathrm{~b}$ & $57,36 \mathrm{a}$ \\
H13-30 & $3,75 \mathrm{~b} \mathrm{c}$ & $7,12 \mathrm{~b}$ & $55,53 \mathrm{a}$ \\
H13-31 & $3,84 \mathrm{~b} \mathrm{a}$ & $7,16 \mathrm{~b}$ & $60,60 \mathrm{a}$ \\
H13-39 & $2,57 \mathrm{e}$ & $16,54 \mathrm{a}$ & $34,75 \mathrm{c}$ \\
L-ChNg1 & $3,26 \mathrm{dc}$ & $15,20 \mathrm{a}$ & $45,58 \mathrm{~b}$ \\
L-ChNg2 & $2,94 \mathrm{ed}$ & $8,15 \mathrm{~b}$ & $40,09 \mathrm{cb}$ \\
DMSH & 0,57 & 4,77 & 9,45 \\
\hline CE & & & \\
\hline 2,0 & $3,36 \mathrm{a}$ & $7,89 \mathrm{~b}$ & $45,96 \mathrm{~b}$ \\
2,5 & $3,56 \mathrm{a}$ & $9,17 \mathrm{~b} \mathrm{a}$ & $53,01 \mathrm{a}$ \\
3,0 & $3,58 \mathrm{a}$ & $10,75 \mathrm{a}$ & $52,21 \mathrm{a}$ \\
DMSH & 0,29 & 2,46 & 4,88 \\
\hline
\end{tabular}

FT: Fenoles totales (mg $\left.100 \mathrm{~g}^{-1}\right)$; Li: Licopeno (mg $\left.100 \mathrm{~g}^{-1}\right)$; CA: Capacidad antioxidante (mm TEAC g-1 $)$; CE: Conductividad eléctrica $\left(\mathrm{dS} \mathrm{m}^{-1}\right)$. DMSH: diferencia mínima significativa honesta. ${ }^{\$}$ Medias con la misma letra dentro de la columna no difieren significativamente de acuerdo con la prueba de Tukey $(\mathrm{p} \leq 0,05)$. 
López et al. (2013) para frutos de tomate cherry manejados con variaciones en la concentración de la solución de Steiner de 50 a 100\%. Por su parte, Feltrin et al. (2012) indicaron valores de 5,5 a 7,3 mg $100 \mathrm{~g} \mathrm{~g}^{-1}$ obtenidos con conductividades de $1,5 \mathrm{a}$ $3,5 \mathrm{dS} \mathrm{m}^{-1}$ en la variedad de tomate cherry 'Sweet Million'.

De acuerdo con la comparación de medias (Tabla 5), se observa que entre las líneas experimentales de frutos rojos usados para determinar la concentración de licopeno, se destaca H13-39 con valores de 16,54 mg $100 \mathrm{~g}^{-1}$, sin embargo, ésta no superó al genotipo L-ChNg1 el cual mostró un comportamiento similar $(15,20$ $\mathrm{mg} 100 \mathrm{~g}^{-1}$ ) (Tabla 5), siendo el material de fruto amarillo (H13-26) el que presentó el nivel más bajo de éste pigmento (1,38 mg $\left.100 \mathrm{~g}^{-1}\right)$; este incremento podría estar asociado con estrés salino causado por el gradiente osmótico durante el proceso de maduración (Elia et al. 2000). Los resultados obtenidos son similares a lo reportado para este tipo de tomate por Kavitha et al. (2014) con 7,0 $\mathrm{mg} 100 \mathrm{~g}^{-1}$ peso fresco. Sin embargo, no superó a lo indicado por Pandurangaiah et al. (2016) para la línea elite de germoplasma IIHR-249-1 con diferentes grados de madurez, con valores de 3 a $20 \mathrm{mg} 100 \mathrm{~g}^{-1}$ peso fresco, donde el valor máximo correspondió a un grado de madurez similar al utilizado en este estudio ( $\geq 90 \%$ de coloración de fruto). Adicionalmente, otros autores como Kavitha et al. (2014) también reportaron valores superiores ( $25 \mathrm{mg}$ peso fresco) para ésta misma línea. Es importante señalar que este tipo de pigmento se encuentra en concentraciones bajas o nulas en frutos inmaduros (verdes) o de color amarillo (Pandurangaiah et al., 2016), contrario a lo que sucede con frutos con pigmentación negra, rosa y roja, cuyos contenidos pueden variar entre 10,2 y 11,7 mg $100 \mathrm{~g}^{-1}$ de peso fresco o 26,1 a 76,7 mg $100 \mathrm{~g}^{-1}$ de peso seco (Bhandari et al., 2016; Pandurangaiah et al., 2016). Es por ello que H13-39 y L-ChNg1, son materiales susceptibles a ser considerados dentro de programas de mejoramiento genético enfocados a la obtención de genotipos cherry con altos niveles de licopeno (Ceballos-Aguirre et al., 2012).

Capacidad antioxidante (CA). De manera similar a lo ocurrido con la concentración de FT y $\mathrm{VC}$, la CA presentó variación $(\mathrm{P} \leq 0,05)$ como respuesta a la modificación de la $\mathrm{CE}$ de la solución nutritiva, cuyos valores estuvieron entre 45,96 y 53,01 mm TEAC $\mathrm{g}^{-1}$ (Tabla 5). Con respecto a estos resultados, Bhandari et al. (2016) indicaron para nueve cultivares comerciales de tomate cherry una media de $46,3 \mathrm{mmol} \mathrm{TE} \mathrm{kg}^{-1}$ de peso seco, con un rango de 34,9 a 54,2; éstos mismos autores señalaron para líneas de germoplasma un dato promedio de 54,5, con un rango de valores que superó a las variedades comerciales (40,8 a 74,5 mmol TE kg-1 de peso seco). Sin embargo, también existen reportes donde la modificación en los valores de la conductividad eléctrica $(3,0$, 6,0 y 9,0 dS m ${ }^{-1}$ ) de la solución no afectó de manera significativa el comportamiento de la capacidad antioxidante (508,1 a 627,4 mol TEAC $100 \mathrm{~g}^{-1}$ ) en frutos de tomate cherry 'Mascote' (Bertoldi et al., 2008); variaciones que de acuerdo con FigueroaCares et al. (2018) pueden asociarse a la variedad, condiciones de manejo agronómico y el grado de madurez durante su determinación.

Con excepción del genotipo H13-39, los materiales de fruto de color amarillo y rojo destacaron por su mayor nivel de capacidad antioxidante, con valores que fluctuaron entre 55,5 a 60,60 $\mathrm{mm} \mathrm{TEAC} \mathrm{g}^{-1}$ de PF (Tabla 5); donde de acuerdo con Fanasca et al. (2006), LeivaBrondo et al. (2012) y Pandurangaiah et al. (2016) el fruto de tomate siempre se ha caracterizado por ser fuente de alta concentración de antocianinas (licopeno, luteína y $\beta$-caroteno), ácido ascórbico, vitaminas y compuestos polifenolicos, que en su conjunto interactúan entre sí, dando como resultado la disminución en la actividad de diversos compuestos oxidativos. En este sentido, Noor-Atiqah et al. (2014) señalaron que los frutos con tonalidades amarillas presentan mayores niveles de actividad antioxidante con respecto a los de color negro y rojo, y conforme avanza el grado de madurez la actividad antioxidante también se incrementa (Ceballos-Aguirre et al., 2012; Pandurangaiah et al., 2016). Por otro lado, valores de capacidad antioxidante menores fueron reportadas por Kavitha et al. (2014) $(8,83$ y 28,56 mg AEAC $100 \mathrm{~g}^{-1}$ ) en frutos de trece líneas de tomate cherry.

\section{CONCLUSIONES}

El uso de diferentes niveles de conductividad eléctrica de la solución nutritiva de Steiner no afectó el comportamiento del rendimiento, NFPR, PPF, color, SST, AT y VC; sin embargo, se observó un incremento en el contenido de licopeno y capacidad antioxidante, características que a su vez se encuentran ampliamente vinculadas con el cuidado de la salud contra el daño causado por diversas enfermedades neurodegenerativas, cardiovasculares y algunos tipos de cáncer.

\section{LITERATURA CITADA}

AOAC. 1990. Official Methods of Analysis. 14th ed. Association of Official Analytical Chemists (AOAC), Arlington, Virginia., USA. 
Beckles, D.M. 2012. Factors affecting the postharvest soluble solids and sugar content of tomato (Solanum lycopersicum L.) fruit. Postharvest Biology and Technology 63(1):129-140. doi:10.1016/j. postharvbio.2011.05.016.

Bertoldi, F.C., E.S. Sant'Anna, J.L. BarcelosOliveira, and R. Simoni. 2008. Antioxidant properties of hydroponic cherry tomato cultivated in desalinized wastewater. Acta Hortic. 843:197-202. doi:10.17660/ ActaHortic.2009.843.25.

Bhandari, S.R., M.C. Cho, and J.G. Lee. 2016. Genotypic variation in carotenoid, ascorbic acid, total phenolic, and flavonoid contents, and antioxidant activity in selected tomato breeding lines. Hortic., Enviro Biotech. 57(5):440-452. doi:10.1007/s13580-016-0144-3.

Boada-Higuera, M.Y., J.L. Mejía-Ramírez, N. Ceballos-Aguirre, y F.J. Orozco. 2010. Evaluación agronómica de treinta introducciones de tomate silvestre tipo cereza (Solanum lycopersicum L.). Agronomía 18(2):59-67.

Boches, P., B. Peterschmidt, and J.R. Myers. 2011. Evaluation of a subset of the Solanum lycopersicum var. cerasiforme core collection for horticultural quality and fruit phenolic content. HortScience 46(11):1450-1455.

Carrillo-Rodríguez, J.C., H. López-Mendoza, J.L. Chávez-Servia, E., Rodríguez-Guzmán, P. Sánchez-Peña, and R. Lobato-Ortiz. 2012. Phenotypic divergence on growth and productivity of wild and semi-domesticated cherry tomato grown under greenhouse conditions. Acta Hortic. 947:375-380. doi:10.17660/ActaHortic.2012.947.48.

Carrillo-Rodríguez, J.C., y J.L. Chávez-Servia. 2010. Caracterización agromorfológica de muestras de tomate de Oaxaca. Rev. Fitotec. Mex. 33(4):1-6.

Ceballos, A.N., y C.F.A. Vallejo. 2012. Evaluating the fruit production and quality of cherry tomato (Solanum lycopersicum var. cerasiforme). Rev Fac. Nac. Agron. Medellín 65(2):6593-6604.

Ceballos-Aguirre, N., F.A. Vallejo-Cabrera, y N. Arango-Arango. 2012. Evaluación del contenido de antioxidantes en introducciones de tomate tipo cereza (Solanum spp.). Acta Agron. 61(3):230-238.

Choi, K., G. Lee, Y.J. Han, and J.M. Bunn. 1995. Tomato maturity evaluation using color image analysis. Transactions of the ASAE 38(1):171-176. doi:10.13031/2013.27827
Cruz-Crespo, E., and M. Sandoval-Villa. 2011. Effect of the nutrient solution concentration and substrates mixture on the quality of tomato. Acta Hortic. 947:197-202. doi:10.17660/ActaHortic.2012.947.24.

Csambalik, L., A. Divéky-Ertsey, Z. Pap, C. Orbán, M.M. Stégerné, A. Gere, et al. 2014. Coherences of instrumental and sensory characteristics: case study on cherry tomatoes. J. Food Sci. 79(11):2192-2202. doi:10.1111/1750-3841.12685.

Elia, A., F. Serio, A. Parente, P. Santamaria, and G.R. Rodríguez. 2000. Electrical conductivity of nutrient solution, plant growth and fruit quality of soilless grown tomato. Acta Horticulturae 559:503-508. doi: 10.17660/ ActaHortic.2001.559.73.

Fanasca, S., G. Colla, G. Maiani, E. Venneria, Y. Rouphael, E. Azzini, et al. 2006. Changes in antioxidant content of tomato fruits in response to cultivar and nutrient solution composition. J. Agric. Food Chem. 54(12):4319-4325. doi:10.1021/jf0602572.

Feltrin, V.P., F.C. Bertoldi, M.Shibata, V.M. Rizelio, J.L. Barcelos-Oliveira, and E.S. Sant'Anna. 2012. The ionic concentration influences of nutrient solutions on the physico-chemical characteristics and productivity of two cherry tomato cultivars cultivated in NFT hydroponic system. Acta Hortic. 947:269-276. doi:10.17660/ActaHortic.2012.947.34.

Fernandes, C., J.E. Corá, y L.T. Braz. 2007. Classificação de tomate-cereja em função do tamanho e peso dos frutos. Hortic. Bras. 25:275-278. doi:10.1590/S010205362007000200029 .

Figueroa-Cares, I.E., O. Cruz-Álvarez, M.T. Martínez-Damián, J.E. Rodríguez-Pérez, M.T. Colinas-León, y S. Valle-Guadarrama. 2018. Calidad nutricional y capacidad antioxidante en variedades y genotipos nativos de jitomate (Solanum lycopersicum L.). Rev. Fac. Agron. (LUZ) 35(1):63-84.

FIRA. 2016. Fideicomisos Instituidos en Relación con la Agricultura (FIRA). 2016. Panorama agroalimentario: Tomate rojo 2016. Disponible en https://www.gob.mx/cms/ uploads/attachment/file/200635/Panorama_ Agroalimentario_Tomate_Rojo_2016.pdf (Consulta 12 septiembre 2017).

Guil-Guerrero, J.L., and M.M. Rebolloso-Fuentes. 2009. Nutrient composition and antioxidant activity of eight tomato (Lycopersicon esculentum) varieties. J. Food Compos. Anal. 22(2):123-129. doi:10.1016/j.jfca.2008.10.012. 
Inbaraj, B.S., H. Lu, C.F. Hung, W.B. Wu, C.L. Lin, and B.H. 2008. Determination of carotenoids and their esters in fruits of Lycium barbarum Linnaeus by HPLC-DAD-APCI-MS. J. Pharm. Biomed. Anal. 47(4-5):812-818. doi:10.1016/j.jpba.2008.04.001

Jacota, S.K., and H.M. Dani. 1982. A new colorimetric technique for the estimation of vitamin $\mathrm{C}$ using folin phenol reagent. Anal. Biochem. 127:178-182. doi:10.1016/00032697(82)90162-2

Juárez-López, P., R. Medina-Torres, E. CruzCrespo, D. W. Reed, M. Kent, L. CisnerosZevallos, et al. 2013. Effect of electrical conductivity of the nutrient solution on fruit quality of three native tomato genotypes (Lycopersicon esculentum var. cerasiforme). Acta Hortic. 1034:505-508. doi:10.17660/ ActaHortic.2014.1034.63.

Kavitha, P., K.S. Shivashankara, V.K. Rao, A.T. Sadashiva, K.V. Ravishankar, and G.J. Sathish. 2014. Genotypic variability for antioxidant and quality parameters among tomato cultivars, hybrids, cherry tomatoes and wild species. J. Sci. Food Agr. 94(5):993999. doi:10.1002/jsfa.6359.

Kim, H.J., J.M. Fonseca, C. Kubota, M. Kroggel, and J.H. Choi. 2008. Quality of fresh-cut tomatoes as affected by salt content in irrigation water and post-processing ultraviolet-C treatment. J. Sci. Food Agr. 88:1969-1974. doi:10.1002/ jsfa.3305.

Krauss, S., W.H. Schnitzler, and M. Woitk. 2006. Fruit quality characteristics of tomatoes at different EC values in a simplified recirculating soilless. Acta Hortic. 747:457463. doi:10.17660/ActaHortic.2007.747.57.

Leiva-Brondo, M., M. Valcárcel, C. Cortés-Olmos, S. Roselló, J. Cebolla-Cornejo, and F. Nuez. 2012. Exploring alternative germplasm for the development of stable high vitamin $\mathrm{C}$ content in tomato varieties. Scientia Horticulturae 133:84-88. doi:10.1016/j. scienta.2011.10.013.

Minolta. 1994. Precise Color Communication. Minolta Corporation Instrument System Division. New Jersey, USA.

Noor-Atiqah, A.A.K., A. M. Maisarah, and R. Asmah. 2014. Comparison of antioxidant properties of tamarillo (Cyphomandra betacea), cherry tomato (Solanum lycopersicum var. cerasiforme) and tomato (Lycopersicon esculentum). Int. Food Res. J. 21(6):2355-2362.
Ozgen, M., N.R. Reese, Z.A., Tulio, C.J. Scheerens, and R.A. Miller. 2006. Modified 2,2-Azinobis-3-ethylbenzothiazoline-6-sulfonic acid (ABTS) method to measure antioxidant capacity of selected small fruits and comparison to ferric reducing antioxidant power (FRAP) and 2,2'-diphenil-1picrylhydrazyl (DPPH). J. Agric. Food Chem. 54(4):1151-1157. doi:10.1021/jf051960d.

Pandurangaiah, S., K.V. Ravishankar, K.S. Shivashankar, A.T. Sadashiva, K. Pillakenchappa, and S.K. Narayanan. 2016. Differential expression of carotenoid biosynthetic pathway genes in two contrasting tomato genotypes for lycopene content. J. Biosci. 41(2):257-264. doi: 10.1007/ s12038-016-9602-4.

Parra-Gómez, M.A., R. Lobato-Ortiz, J.J. GarcíaZavala, D. Reyes-López y M.J. VelascoAlvarado. 2016. Evaluación de líneas de una cruza interespecífica de tomate. Rev. Fitotec. Mex. 39(1):59-65.

Preczenhak, A.P., J.T. Resende, R. Chagas, P.R. Silva, and K. Schwarz. 2014. Caracterização agronômica de genótipos de minitomate. Hortic. Bras. 32(3):348-356. doi:10.1590/ S0102-053620140003000018.

Ramya, R., M. Ananthan, and V. Krishnamoorthy. 2016. Evaluation of cherry tomato [Solanum lycopersicum L. var. cerasiforme (Dunnal) A. Gray] genotypes for yield and quality traits. Asian J. Hortic. 11(2):329-334. doi:10.15740/ HAS/TAJH/11.2/329-334.

Ranc, N., S. Muños, and S. Santoni. 2008. A clarified position for Solanum lycopersicum var. cerasiforme in the evolutionary history of tomatoes. BMC Plant Biol. 8(1):1-16. doi:10.1186/1471-2229-8-130.

Rodríguez, E., D. Vargas, J.J. Sánchez, R. Lepiz, A. Rodríguez, J.A. Ruiz, et al. 2009. Etnobotánica de Solanum lycopersicum var. cerasiforme en el Occidente de México. Nat. Desarro. 7:46-59.

Sadler, G., J. Davis, and D. Dezman. 1990. Rapid extraction of lycopene and carotene from reconstituted tomato paste and pink grapefruit homogenates. J. Food Sci. 55(5):1460-1461. doi:10.1111/j.1365-2621.1990. tb03958.x.

SAS. 2002. SAS/STAT user's guide: Statistics. Ver. 9.00. 1503 p. SAS Institute Inc., Cary, North Caroline, USA. 
SIAP. 2016. Sistema de Información y Estadística Agroalimentaria y Pesquera (SIAP). 2016. Anuario estadístico de la producción agrícola. Secretaria de Agricultura, Ganadería, Desarrollo Rural y Pesca (SAGARPA). México [en línea]. Disponible en http://www.gob. $\mathrm{mx} /$ siap/acciones-y-programas/produccionagricola-33119?idiom=es (Consulta 10 septiembre 2017).

Steiner, A.A. 1984. The universal nutrient solution. p. 633-650. In International Society for Soilless Culture (ed.). Proceedings 6th International Congress on Soilles Culture. $1^{\text {st }}$. ed. Secretariat of ISOSC, Wageningen, the Netherlands.
Wang, Y.T., S.H. Huang, R.L. Liu, and J.Y. Jin. 2007. Effects of nitrogen application on flavor compounds of cherry tomato fruits. J. Plant Nutrition Soil Science 170(4):461-468. doi:10.1002/jpln.200700011.

Waterman, P.G., and S. Mole. 1994. Analysis of phenolic plant metabolites. Blackwell Scientific Publications, Oxford, UK. 